\title{
Identification of biomarkers and pathways of mouse embryonic fibroblasts with the dysfunction of mitochondrial DNA
}

Hanming $\mathrm{Gu}^{1,2, *}$

${ }^{1}$ School of Electronic, Information and Electrical Engineering, Shanghai Jiao Tong University, Shanghai, China

${ }^{2}$ SHU-UTS SILC Business School, Shanghai University, Shanghai, China

"Corresponding author: Hanming Gu, Information and Electrical Engineering, Shanghai Jiao Tong University, Shanghai, China

laygmp@gmail.com 


\section{Abstract}

Mitochondrial diseases are clinically heterogeneous which involve multiple systems such as organs that are highly dependent on metabolism. Dysfunction of mtDNA is the main cause of mitochondrial diseases that trigger inflammation and immune responses. Here, we aim to identify the biological function and pathways of MEFs with the dysfunction of mtDNA through deletion of YME1L. The gene expression profiles of GSE161735 dataset were originally created by the Illumina NovaSeq 6000 (Mus musculus) for gene biogenesis and function panel. The biological and functional pathways were analyzed by the Kyoto Encyclopedia of Genes and Genomes pathway (KEGG), Gene Ontology (GO), and Reactom visual map. KEGG and GO results showed the metabolism and immune responses were mostly affected by the loss of mtDNA. Moreover, we discovered several interacting genes including POLR2F, HIST1H2BJ, PPP1CC, HOXB4, ARG1, APITD1, BUB1B, POLR2K, HOXC4, and HOXB3 were involved in the regulation of metabolic or cancer diseases. Further, we predicted several regulators that had the ability to affect mitochondria during the dysfunction of mtDNA by L1000fwd analysis. Thus, this study provides further insights into the mechanism of mtDNA in metabolic diseases.

\section{Introduction}

Mitochondria are critical regulators of metabolism in various cellular pathways, including fatty acid oxidation, oxidative phosphorylation, Krebs cycle, urea cycle, and gluconeogenesis ${ }^{1}$. Mitochondria also play a crucial role in other cellular processes such as amino acid metabolism, lipid metabolism, calcium homeostasis, and apoptosis ${ }^{2,3}$. Mitochondrial diseases are complicated and involve mitochondrial and nuclear DNA mutations ${ }^{4}$. Generally, they cause the main defect in oxidative phosphorylation that involves ATP production, but lack of other enzymes such as Krebs enzymes also can lead to severe diseases ${ }^{5-7}$.

Mitochondrial DNA (mtDNA) encodes crucial proteins of the oxidative phosphorylation system $^{8}$. This system contains an electron transport chain and ATP synthase, which makes mitochondrial respiration and ATP production ${ }^{9}$. Recently, it has been shown that mitochondrial DNA has various functions in immune responses ${ }^{10}$. Numerous evidence 
was identified for mtDNA regulating immune regulation ${ }^{11}$. Firstly, mtDNA is small and encodes 13 oxidative phosphorylation mRNAs, tRNAs, and ribosomal RNAs which are needed for mitochondrial matrix constitution ${ }^{12}$. Secondly, mtDNA copy number is controlled by cell-specific mechanisms and in response to various environmental stresses $^{13}$. Thirdly, mtDNA contains unique bacterial nucleic acid sequences that make it more like foreign $D_{N A}{ }^{14}$. Finally, mtDNA may exhibit persistent oxidative damage modifications or mutations due to its oxidative environment and DNA repair mechanisms $^{15}$. Thus, mtDNA may have an essential role in innate immune response and inflammation.

In this study, we investigated the block of mtDNA in MEFs by using the protease YME1L. YME1L preserves nucleotide by supporting de novo nucleotide synthesis and limiting mitochondrial nucleotide transport and accumulation ${ }^{16,17}$. We identified DEGs, candidate regulators, and the biological process in YME1L KO MEFs by performing a comprehensive bioinformatics analysis. The functional enrichment analysis and proteinprotein interaction were utilized for discovering the significant gene nodes. These key genes and signaling pathways may be essential to therapeutic interventions of mitochondrial diseases.

\section{Methods}

\section{Data resources}

The GSE161735 dataset was downloaded from the GEO database (http://www.ncbi.nlm.nih.gov/geo/). It was produced by Illumina NovaSeq 6000 (Mus musculus) for cell biogenesis and function panel, Max Planck Institute for Biology of Ageing, Cologne, Germany. RNA-Seq analysis was performed using mouse embryonic fibroblasts (MEFs) with the knockout of YME1L gene (YME1L KO) as well as wild-type (WT) controls.

Data acquisition and preprocessing

The GSE161735 dataset that contains gene expression related to gene functions from the MEFs with the knockout of YME1L gene (YME1L KO) was conducted by $R \operatorname{script}^{18,}$ 
19. We used a classical t-test to identify DEGs with $P<0.01$ and fold change $\geq 1.5$ as being statistically significant.

Gene functional analysis

Gene Ontology (GO) is a structured bioinformatics resource that includes three domains: biological processes (BP), cellular components (CC), and molecular functions (MF). Kyoto Encyclopedia of Genes and Genomes (KEGG) database is the pathway tool that integrates functional information and the known and unknown biological pathways. GO and KEGG pathways were conducted by utilizing the Database for Annotation, Visualization, and Integrated Discovery (DAVID) (http://david.ncifcrf.gov/) and Reactome (https://reactome.org/) ${ }^{20}$. $\mathrm{P}<0.05$ was considered statistically significant.

Module analysis

We study the connected regions in protein-protein interaction (PPI) networks using the Molecular Complex Detection (MCODE) of Cytoscape software ${ }^{21,22}$. The significant clusters were selected from the constructed PPI network using MCODE and String database (https://string-db.org/). The pathway enrichment analyses were performed by using Reactome, and $\mathrm{P}<0.05$ was used as the cutoff criterion.

Reactome analysis

Reactome pathway (https://reactome.org/) was conducted to obtain the visualization analysis of potential pathways. $\mathrm{P}<0.05$ was considered statistically significant.

\section{Results}

Identification of DEGs of Yme1I KO MEFs in comparison to those of WT MEFs Mitochondrial DNA (mtDNA) elicits a type I interferon response, but the mechanism of mtDNA release remains unknown. The mitochondrial protease YME1L maintains nucleotide pools by supporting de novo nucleotide synthesis and the accumulation of mtDNA. Transcript profiles of WT and Yme1l KO MEFs were analyzed by differentially expressed genes (DEGs) from the Max Planck Institute for Biology of Ageing, Germany. A total of 3495 genes were identified to be differentially expressed in Yme1l KO MEFs 
with the threshold of $P<0.05$. The top 10 up-and down-regulated genes are listed in Table 1.

KEGG analysis of DEGs of Yme1I KO MEFs in comparison to those of WT MEFs To identify the biological functions and pathways of DEGs of Yme1l KO MEFs, we conducted a KEGG pathway enrichment analysis (Supplemental Table S1). KEGG pathway is a dataset collection for analyzing molecular interaction and relation networks. Our study showed the top ten enriched KEGG pathways including "Metabolic pathways", "MAPK signaling pathway", "Herpes simplex infection", "Parkinson's disease", "Huntington's disease", "Oxidative phosphorylation", "Autoimmune thyroid disease", "Leishmaniasis", "Metabolism of xenobiotics by cytochrome P450", and "Antigen processing and presentation" (Figure 1).

GO analysis of DEGs from Yme1l KO MEFs

Gene Ontology (GO) analysis is a useful tool for sorting genes, which contains cellular components (CC), molecular functions (MF), and biological processes (BP). We identified the top ten cellular components including "cytoplasm", "extracellular exosome", "extracellular space", "mitochondrion", "axon", "mitochondrial inner membrane", "respiratory chain", "endoplasmic reticulum exit site", "mitochondrial respiratory chain complex III", and "MHC class II protein complex" (Figure 1). We also identified the top ten biological processes: "oxidation-reduction process", "multicellular organism development", "immune system process", "aging”, "anterior/posterior pattern specification", "spleen development", "embryonic skeletal system development", "antigen processing and presentation", "embryonic skeletal system morphogenesis", and "antigen processing and presentation of exogenous peptide antigen via $\mathrm{MHC}$ class II" (Figure 1). We then identified the top ten molecular functions: "protein homodimerization activity", "oxidoreductase activity", "transcription factor activity, sequence-specific DNA binding", "receptor binding", "sequence-specific DNA binding", "peptide antigen binding", "T cell receptor binding", "high voltage-gated calcium channel activity", "beta-2-microglobulin binding", and "TAP binding" (Figure 1 and Supplemental Table S1). 
PPI network and Module analysis

PPI networks were created to analyze the relationships of DGEs at the protein level.

The criterion of combined score $>0.7$ was conducted and the PPI network was constructed by using 301 nodes and 627 interactions. Among these nodes and edges, the top ten genes with the highest scores are shown in Table 2. The significant modules of DEGs of Yme1I KO MEFs in comparison to those of WT MEFs were selected to show the functional annotation (Figure 2).

Reactome Pathway analysis

To further understand the potential functions of DEGs, we also identified the signaling pathways by using Reactome Pathway Database. The top ten signaling pathways: "Response of EIF2AK1 (HRI) to heme deficiency", "Activation of anterior HOX genes in hindbrain development during early embryogenesis", "Activation of HOX genes during differentiation", "ATF4 activates genes in response to endoplasmic reticulum stress", "PERK regulates gene expression", "Defective Base Excision Repair Associated with OGG1", "Response of EIF2AK4 (GCN2) to amino acid deficiency", "Respiratory electron transport", "EPHA-mediated growth cone collapse", and "Rhesus blood group biosynthesis" (Supplemental Table S2). We then constructed the visual reaction map according to the signaling pathways (Figure 3).

Potential regulators analysis

To further know the potential regulators, we use the L1000 fireworks display system that can predict bioactive molecules. The system indicated the potential pathways that may be inhibited. We selected the top ten molecules according to the DEGs and inhibitor map: "vincristine", "BRD-K89059493", "VU-0410183-2", "indirubin", "heliomycin”, "ATSUMO-1", "PD-98059", “tosyl-phenylalanyl-chloromethyl-ketone”, "loteprednol”, and "BRD-K51556300" (Figure 4 and Supplemental Table S3).

\section{Discussion}


Mitochondria are key scaffolds of cellular metabolism and signaling, but they are also being proved as critical participants in immune responses to pathogens and cellular damage ${ }^{23}$. Mitochondria are not only necessary for antiviral signaling but also the essential sources of endogenous DAMPs ${ }^{11}$. mtDNA triggers pro-inflammatory processes and type I IFN responses owing to its unique structural features and heightened susceptibility to oxidative damage ${ }^{24}$. Thus, future work to disclose the mechanism of mtDNA will have broad implications for knowing mitochondrial diseases, perhaps leading to new avenues for therapy to improve human health.

To understand the effects of mtDNA, we analyzed the gene expressions related to YME1L KO MEFs in this study. By analyzing the DEGs, ten proteins were identified according to the PPI network analysis, which may be important in mitochondrial diseases or other diseases. For example, POLR2F is related to the periventricular white matter hyperintensities (WMH; PVWMH) $)^{25}$. HIST1H2BJ is identified for predicting the prognosis of cervical cancer patients ${ }^{26}$. PPP1CC is required for SLFN11 to cleave tRNAs and further to inhibit the translation ${ }^{27}$. G protein, GPCR, and related proteins are involved in widely physiological and pathophysiological processes ${ }^{28-33}$. PPP1CC plays an important role in regulating the GPCR signaling pathways ${ }^{34}$. HOXB4 can induce the expansion of hematopoietic and promote the malignant progression of ovarian cancer ${ }^{35}$, ${ }^{36}$. Arg1 is related to the tumor-associated macrophage and inflammation resolution ${ }^{37}$. Circadian gene clocks regulate numerous physiological processes such as metabolism, immune, and aging ${ }^{38-41}$. A recent finding is that Arg1 is up-regulated in the Rev-erb KO mice $^{42}$. APITD1 contains a p53-binding domain, which indicates low expression in neuroblastoma tumors ${ }^{43}$. BUB1B is positively correlated with cancer progression and promotes hepatocellular carcinoma through triggering the mTORC1 signaling pathway ${ }^{44}$. POLR2K involves prostate cancer progression and lethal disease ${ }^{45}$. HOXC4 controls prostate cancer cells via regulating genes and binding sites ${ }^{46}$. HBOX 3 is associated with the progression of prostate cancer ${ }^{47}$. Thus, dysfunction of mtDNA may cause potential diseases not only related to mitochondria but other kinds of diseases as well.

KEGG and GO analyses indicated that metabolism and immune were the main pathological processes after blocking the mtDNA. The processes of "Metabolic pathways", "Oxidative phosphorylation", "Metabolism of xenobiotics by cytochrome 
P450" and "oxidation-reduction process" in KEGG and GO were significantly changed. Since mitochondria are the main organelle in cells for energy production, the loss of the function of mtDNA will probably cause metabolic dysfunctions. We also found "Autoimmune thyroid disease", "Antigen processing and presentation" and "immune system process" were involved in the process of mtDNA blocking. Similarly, mtDNA acts as a stress that promotes the antiviral innate immune response ${ }^{48}$. mtDNA activates the innate immune cGAS/STING pathway in the absence of apoptosis, which causes the dying cells to secrete type I interferon ${ }^{49}$. mtDNA also involves the NF-KB signaling pathway that widely regulates the inflammatory factors ${ }^{50-52}$. It was reported that the dysfunction of mitochondria leads to bone defect and bone diseases ${ }^{53}$. Here, we also found the block of mtDNA can affect embryonic skeletal system development and its morphogenesis.

In summary, we discovered the biological pathways in Yme1IKO MEFs by analyzing gene functions. Metabolism and immune response are the mainly triggered pathways during the loss of mtDNA. Future studies will focus on the development of the potential regulators of mitochondrial diseases based on our study. Our study provides further insights into the dysfunction of mtDNA, which may facilitate drug development. 


\section{Reference}

[1] Spinelli JB, Haigis MC: The multifaceted contributions of mitochondria to cellular metabolism. Nat Cell Biol 2018, 20:745-54.

[2] Osellame LD, Blacker TS, Duchen MR: Cellular and molecular mechanisms of mitochondrial function. Best Pract Res Clin Endocrinol Metab 2012, 26:711-23.

[3] Yuan G, Yang S, Liu M, Yang S: RGS12 is required for the maintenance of mitochondrial function during skeletal development. Cell Discov 2020, 6:59.

[4] Taylor RW, Turnbull DM: Mitochondrial DNA mutations in human disease. Nat Rev Genet 2005, 6:389-402.

[5] Kanungo S, Morton J, Neelakantan M, Ching K, Saeedian J, Goldstein A: Mitochondrial disorders. Ann Transl Med 2018, 6:475.

[6] Xu L, Cheng Q, Hua B, Cai T, Lin J, Yuan G, Yan Z, Li X, Sun N, Lu C, Qian R: Circadian gene Clock regulates mitochondrial morphology and functions by posttranscriptional way. bioRxiv 2018:365452.

[7] Cai T, Hua B, Luo D, Xu L, Cheng Q, Yuan G, Yan Z, Sun N, Hua L, Lu C: The circadian protein CLOCK regulates cell metabolism via the mitochondrial carrier SLC25A10. Biochim Biophys Acta Mol Cell Res 2019, 1866:1310-21.

[8] Hebert SL, Lanza IR, Nair KS: Mitochondrial DNA alterations and reduced mitochondrial function in aging. Mech Ageing Dev 2010, 131:451-62.

[9] Kuhlbrandt W: Structure and function of mitochondrial membrane protein complexes. BMC Biol 2015, 13:89.

[10] Riley JS, Tait SW: Mitochondrial DNA in inflammation and immunity. EMBO Rep 2020, 21:e49799.

[11] Weinberg SE, Sena LA, Chandel NS: Mitochondria in the regulation of innate and adaptive immunity. Immunity 2015, 42:406-17.

[12] Mai N, Chrzanowska-Lightowlers ZM, Lightowlers RN: The process of mammalian mitochondrial protein synthesis. Cell Tissue Res 2017, 367:5-20.

[13] Zhao L: Mitochondrial DNA degradation: A quality control measure for mitochondrial genome maintenance and stress response. Enzymes 2019, 45:311-41.

[14] Kubo T, Nishizawa S, Sugawara A, Itchoda N, Estiati A, Mikami T: The complete nucleotide sequence of the mitochondrial genome of sugar beet (Beta vulgaris L.) reveals a novel gene for tRNA(Cys)(GCA). Nucleic Acids Res 2000, 28:2571-6.

[15] Cline SD: Mitochondrial DNA damage and its consequences for mitochondrial gene expression. Biochim Biophys Acta 2012, 1819:979-91.

[16] Campbell CL, Thorsness PE: Escape of mitochondrial DNA to the nucleus in yme1 yeast is mediated by vacuolar-dependent turnover of abnormal mitochondrial compartments. J Cell Sci 1998, 111 ( Pt 16):2455-64.

[17] Stiburek L, Cesnekova J, Kostkova O, Fornuskova D, Vinsova K, Wenchich L, Houstek J, Zeman J: YME1L controls the accumulation of respiratory chain subunits and is required for apoptotic resistance, cristae morphogenesis, and cell proliferation. Mol Biol Cell 2012, 23:1010-23.

[18] Hanming G, Wei W, Gongsheng Y: Identification of potential biomarkers and inhibitors in SARS-CoV2 infected macaques. Research Square 2020.

[19] Gu H, Yuan G: Identification of potential biomarkers and inhibitors for SARS-CoV-2 infection. medRxiv 2020:2020.09.15.20195487.

[20] Yuan G: Identification of biomarkers and pathways of mitochondria in sepsis patients. bioRxiv 2021:2021.03.29.437586.

[21] Gu H, Yuan G: Identification of key genes in SARS-CoV-2 patients on bioinformatics analysis. bioRxiv 2020:2020.08.09.243444. 
[22] Gu H, Wang W, Yuan G: Identification of biomarkers and candidate inhibitors for multiple myeloma. bioRxiv 2021:2021.02.25.432847.

[23] Mohanty A, Tiwari-Pandey R, Pandey NR: Mitochondria: the indispensable players in innate immunity and guardians of the inflammatory response. J Cell Commun Signal 2019, 13:303-18.

[24] West AP, Shadel GS: Mitochondrial DNA in innate immune responses and inflammatory pathology. Nat Rev Immunol 2017, 17:363-75.

[25] Armstrong NJ, Mather KA, Sargurupremraj M, Knol MJ, Malik R, Satizabal CL, Yanek LR, Wen W, Gudnason VG, Dueker ND, Elliott LT, Hofer E, Bis J, Jahanshad N, Li S, Logue MA, Luciano M, Scholz M, Smith AV, Trompet S, Vojinovic D, Xia R, Alfaro-Almagro F, Ames D, Amin N, Amouyel P, Beiser AS, Brodaty H, Deary IJ, Fennema-Notestine C, Gampawar PG, Gottesman R, Griffanti L, Jack CR, Jr., Jenkinson M, Jiang J, Kral BG, Kwok JB, Lampe L, D CML, Maillard P, Marchini J, Bastin ME, Mazoyer B, Pirpamer L, Rafael Romero J, Roshchupkin GV, Schofield PR, Schroeter ML, Stott DJ, Thalamuthu A, Trollor J, Tzourio C, van der Grond J, Vernooij MW, Witte VA, Wright MJ, Yang Q, Morris Z, Siggurdsson S, Psaty B, Villringer A, Schmidt H, Haberg AK, van Duijn CM, Jukema JW, Dichgans M, Sacco RL, Wright CB, Kremen WS, Becker LC, Thompson PM, Mosley TH, Wardlaw JM, Ikram MA, Adams HHH, Seshadri S, Sachdev PS, Smith SM, Launer L, Longstreth W, DeCarli C, Schmidt R, Fornage M, Debette S, Nyquist PA: Common Genetic Variation Indicates Separate Causes for Periventricular and Deep White Matter Hyperintensities. Stroke 2020, 51:2111-21.

[26] Li X, Tian R, Gao H, Yang Y, Williams BRG, Gantier MP, McMillan NAJ, Xu D, Hu Y, Gao Y: Identification of a histone family gene signature for predicting the prognosis of cervical cancer patients. Sci Rep 2017, 7:16495.

[27] Malone D, Lardelli RM, Li M, David M: Dephosphorylation activates the interferon-stimulated Schlafen family member 11 in the DNA damage response. J Biol Chem 2019, 294:14674-85.

[28] Yuan G, Yang S, Gautam M, Luo W, Yang S: Macrophage regulator of G-protein signaling 12 contributes to inflammatory pain hypersensitivity. Annals of Translational Medicine 2021.

[29] Mao SZ, Fan XF, Xue F, Chen R, Chen XY, Yuan GS, Hu LG, Liu SF, Gong YS: Intermedin modulates hypoxic pulmonary vascular remodeling by inhibiting pulmonary artery smooth muscle cell proliferation. Pulm Pharmacol Ther 2014, 27:1-9.

[30] Zhu Z, Xu L, Cai T, Yuan G, Sun N, Lu C, Qian R: Clock represses preadipocytes adipogenesis via GILZ. J Cell Physiol 2018, 233:6028-40.

[31] Yuan G, Yang S, Yang S, Ng A, Oursler MJ: RGS12 is a critical proinflammatory factor in the pathogenesis of inflammatory arthritis via acting in Cox2-RGS12-NF kappa B pathway activation loop. J Bone Miner Res: WILEY 111 RIVER ST, HOBOKEN 07030-5774, NJ USA, 2019. pp. 147-.

[32] Fu C, Yuan G, Yang ST, Zhang D, Yang S: RGS12 Represses Oral Cancer via the Phosphorylation and SUMOylation of PTEN. J Dent Res 2020:22034520972095.

[33] Fan XF, Wang XR, Yuan GS, Wu DH, Hu LG, Xue F, Gong YS: [Effect of safflower injection on endoplasmic reticulum stress-induced apoptosts in rats with hypoxic pulmonary hypertension]. Zhongguo Ying Yong Sheng Li Xue Za Zhi 2012, 28:561-7.

[34] Camp ND, Lee KS, Wacker-Mhyre JL, Kountz TS, Park JM, Harris DA, Estrada M, Stewart A, WolfYadlin A, Hague C: Individual protomers of a $G$ protein-coupled receptor dimer integrate distinct functional modules. Cell Discov 2015, 1.

[35] Li N, Gou JH, Xiong J, You JJ, Li ZY: HOXB4 promotes the malignant progression of ovarian cancer via DHDDS. BMC Cancer 2020, 20:222.

[36] Antonchuk J, Sauvageau G, Humphries RK: HOXB4-induced expansion of adult hematopoietic stem cells ex vivo. Cell 2002, 109:39-45.

[37] Arlauckas SP, Garren SB, Garris CS, Kohler RH, Oh J, Pittet MJ, Weissleder R: Arg1 expression defines immunosuppressive subsets of tumor-associated macrophages. Theranostics 2018, 8:5842-54. 
[38] Zhu Z, Hua B, Shang Z, Yuan G, Xu L, Li E, Li X, Sun N, Yan Z, Qian R, Lu C: Altered Clock and Lipid Metabolism-Related Genes in Atherosclerotic Mice Kept with Abnormal Lighting Condition. Biomed Res Int 2016, 2016:5438589.

[39] Yuan G, Hua B, Cai T, Xu L, Li E, Huang Y, Sun N, Yan Z, Lu C, Qian R: Clock mediates liver senescence by controlling ER stress. Aging 2017, 9:2647-65.

[40] Yuan G, Hua B, Yang Y, Xu L, Cai T, Sun N, Yan Z, Lu C, Qian R: The Circadian Gene Clock Regulates Bone Formation Via PDIA3. J Bone Miner Res 2017, 32:861-71.

[41] Zhu Z, Hua B, Xu L, Yuan G, Li E, Li X, Sun N, Yan Z, Lu C, Qian R: CLOCK promotes 3T3-L1 cell proliferation via Wnt signaling. IUBMB Life 2016, 68:557-68.

[42] Zhang T, Chen M, Guo L, Yu F, Zhou C, Xu H, Wu B: Reverse Erythroblastosis Virus alpha Antagonism Promotes Homocysteine Catabolism and Ammonia Clearance. Hepatology 2019, 70:1770-84.

[43] Krona C, Ejeskar K, Caren H, Abel F, Sjoberg RM, Martinsson T: A novel 1p36.2 located gene, APITD1, with tumour-suppressive properties and a putative p53-binding domain, shows low expression in neuroblastoma tumours. Br J Cancer 2004, 91:1119-30.

[44] Qiu J, Zhang S, Wang P, Wang H, Sha B, Peng H, Ju Z, Rao J, Lu L: BUB1B promotes hepatocellular carcinoma progression via activation of the mTORC1 signaling pathway. Cancer Med 2020, 9:8159-72.

[45] Kelly RS, Sinnott JA, Rider JR, Ebot EM, Gerke T, Bowden M, Pettersson A, Loda M, Sesso HD, Kantoff PW, Martin NE, Giovannucci EL, Tyekucheva S, Heiden MV, Mucci LA: The role of tumor metabolism as a driver of prostate cancer progression and lethal disease: results from a nested case-control study. Cancer Metab 2016, 4:22.

[46] Luo Z, Farnham PJ: Genome-wide analysis of HOXC4 and HOXC6 regulated genes and binding sites in prostate cancer cells. PLoS ONE 2020, 15:e0228590.

[47] Chen J, Zhu S, Jiang N, Shang Z, Quan C, Niu Y: HoxB3 promotes prostate cancer cell progression by transactivating CDCA3. Cancer Lett 2013, 330:217-24.

[48] West AP, Khoury-Hanold W, Staron M, Tal MC, Pineda CM, Lang SM, Bestwick M, Duguay BA, Raimundo N, MacDuff DA, Kaech SM, Smiley JR, Means RE, Iwasaki A, Shadel GS: Mitochondrial DNA stress primes the antiviral innate immune response. Nature 2015, 520:553-7.

[49] McArthur K, Whitehead LW, Heddleston JM, Li L, Padman BS, Oorschot V, Geoghegan ND, Chappaz S, Davidson S, San Chin H, Lane RM, Dramicanin M, Saunders TL, Sugiana C, Lessene R, Osellame LD, Chew TL, Dewson G, Lazarou M, Ramm G, Lessene G, Ryan MT, Rogers KL, van Delft MF, Kile BT: BAK/BAX macropores facilitate mitochondrial herniation and mtDNA efflux during apoptosis. Science 2018, 359.

[50] Berneburg M, Plettenberg H, Krutmann J: Photoaging of human skin. Photodermatol Photoimmunol Photomed 2000, 16:239-44.

[51] Yuan G, Yang S, Ng A, Fu C, Oursler MJ, Xing L, Yang S: RGS12 Is a Novel Critical NF-kappaB Activator in Inflammatory Arthritis. iScience 2020, 23:101172.

[52] Yuan G, Xu L, Cai T, Hua B, Sun N, Yan Z, Lu C, Qian R: Clock mutant promotes osteoarthritis by inhibiting the acetylation of NFkappaB. Osteoarthritis Cartilage 2019, 27:922-31.

[53] Gandhi SS, Muraresku C, McCormick EM, Falk MJ, McCormack SE: Risk factors for poor bone health in primary mitochondrial disease. J Inherit Metab Dis 2017, 40:673-83. 
Figures and Figure Legends

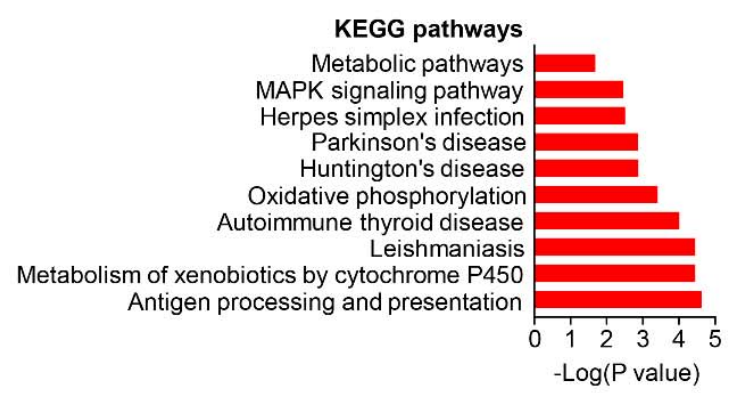

Cellular components

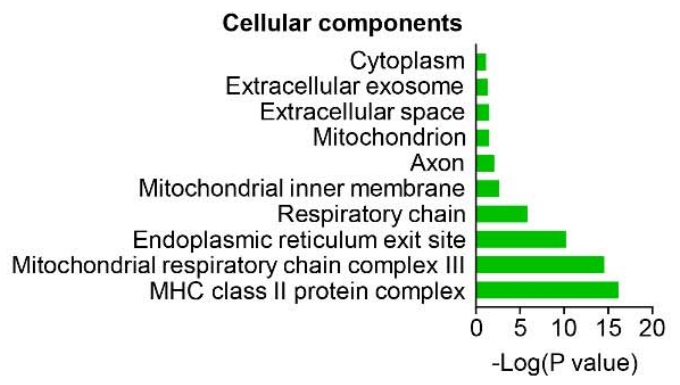

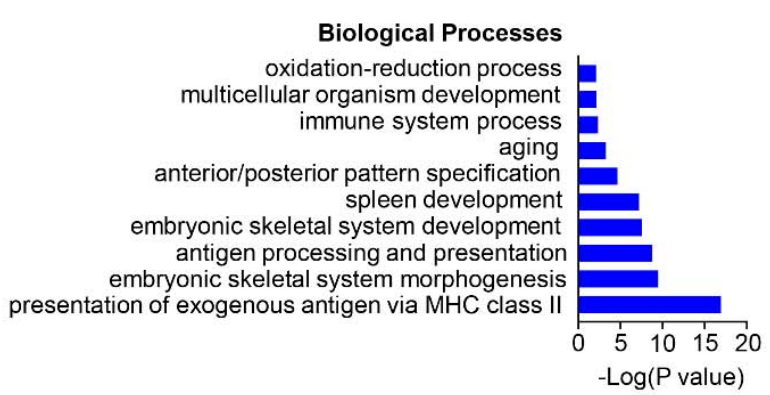

Molecular Functions

Protein homodimerization activity

Oxidoreductase activity

Transcription factor activity, sequence-specific DNA binding

Sequence-specific DNA binding

Peptide antigen binding

High voltage-gated calcium channel activity Beta-2-microglobulin binding TAP binding

\begin{tabular}{lllll}
\hline 0 & 5 & 10 & 15 & 20
\end{tabular} $-\log (P$ value $)$

Figure 1. The KEGG pathways, biological process, cellular component, and molecular function terms enriched by the DEGs. DEGs =differentially expressed genes, KEGG = Kyoto Encyclopedia of Genes and Genomes. 


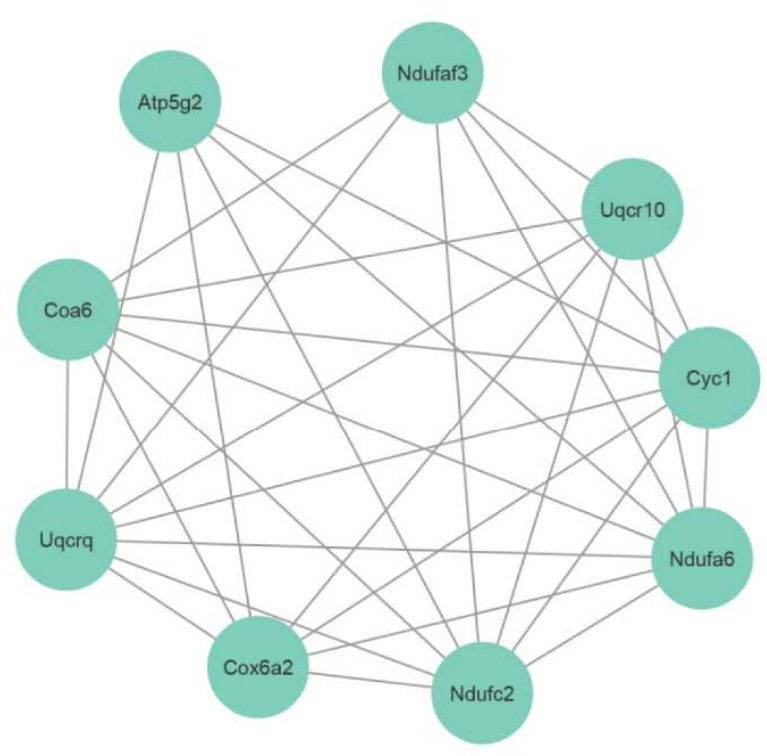

Cluster 1

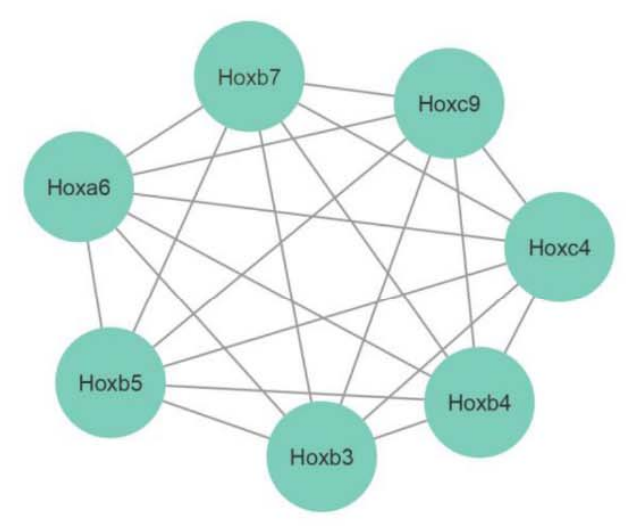

Cluster 2

Figure 2. Top two modules from the PPI network. 


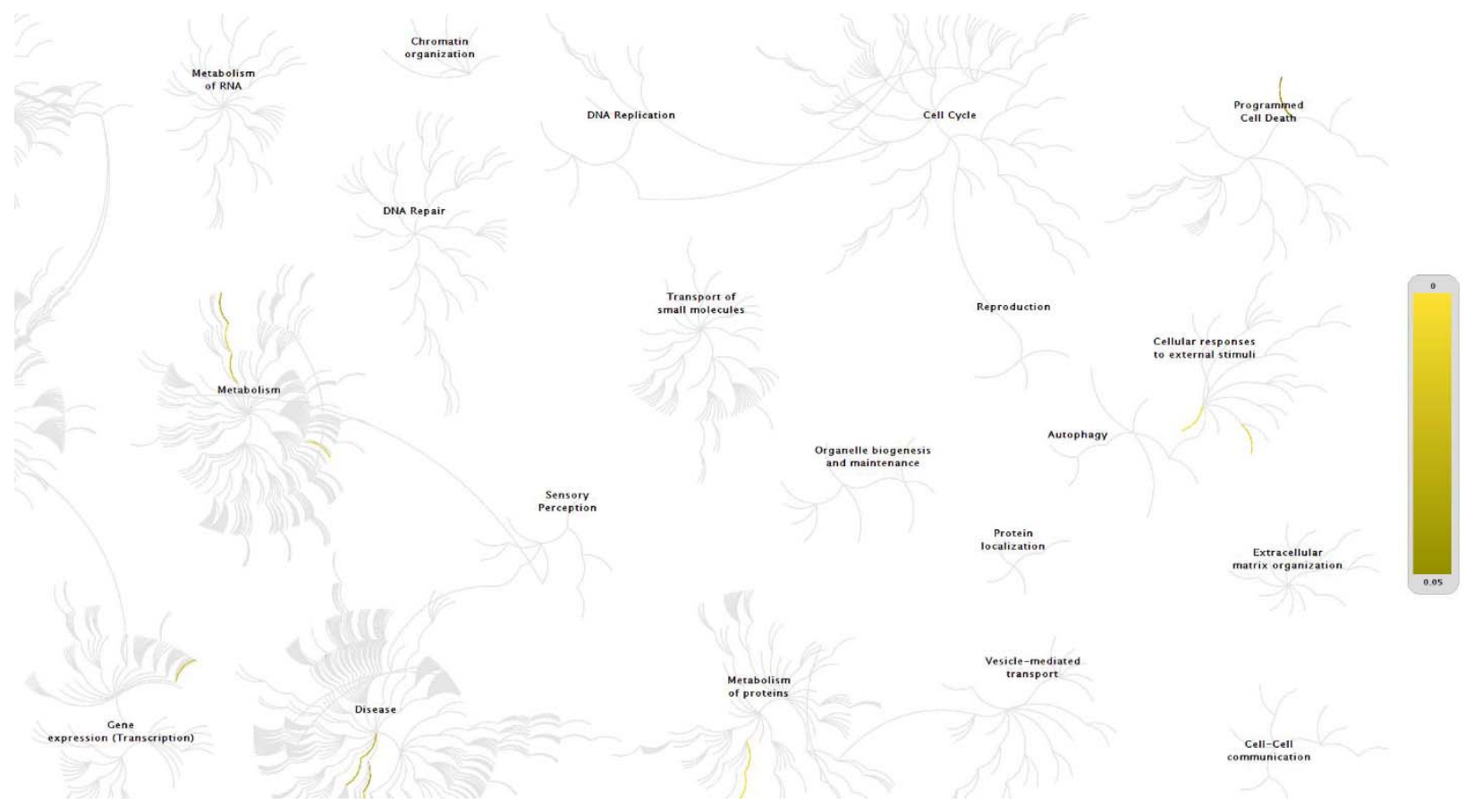

Figure 3. The Reactom pathways. The top significantly changed genes obtained from the GSE161735 dataset $(\mathrm{P}<0.01)$. The yellow color represents the most relevant signaling pathways. 


\section{p-value MOA}

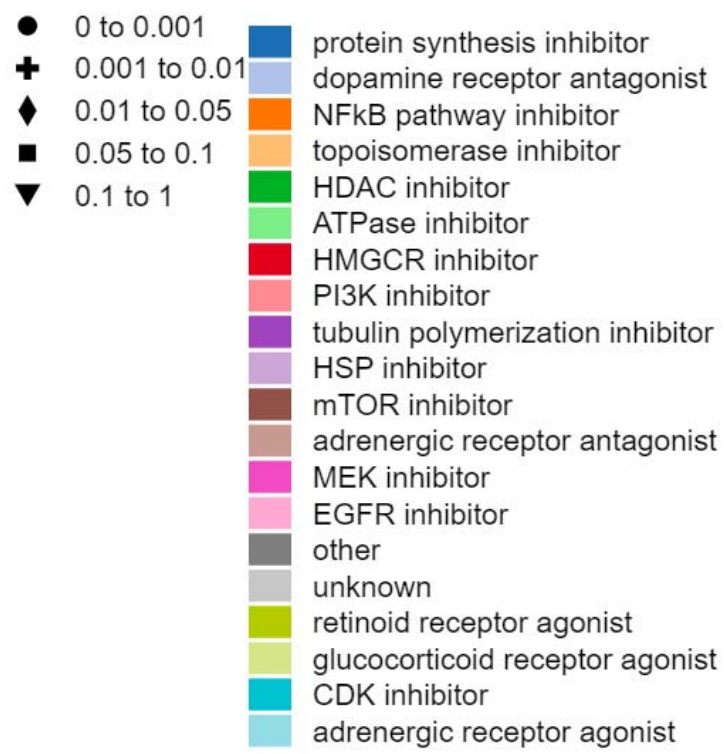

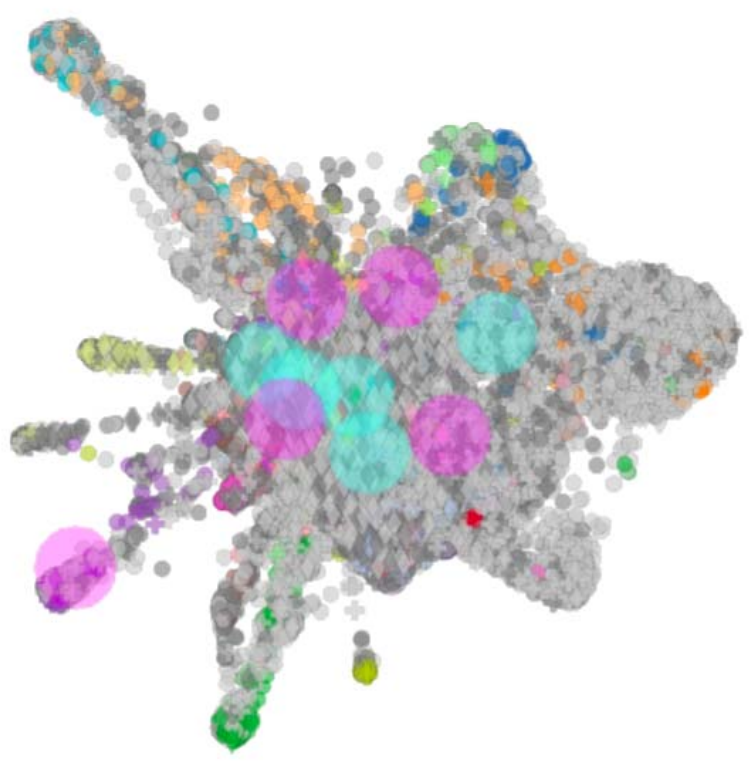

Figure 4. Inhibitor predictions by L1000FDW. The significantly changed genes obtained from the GSE161735 dataset. Dots are the Mode of Action (MOA) of the respective drug. 


\begin{tabular}{lll} 
Table 1 & & \\
\hline Entrez gene & Gene Symble & Regulation \\
\hline \multicolumn{1}{l}{ Top 10 down-regulated DEGs } & \\
102632126 & Gm30280 & Down \\
114607 & Smt3h2-ps4 & Down \\
2329 & Fmo4 & Down \\
100502647 & Gm11583 & Down \\
187103 & AC187103.3 & Down \\
129085 & AC129085.4 & Down \\
154575 & AC154575.1 & Down \\
102636516 & Gm15742 & Down \\
625785 & Gm6623 & Down \\
667170 & Gm8492 & Down \\
Top 10 up-regulated DEGs & \\
2532 & Ackr1 & up \\
92591 & Asb16 & up \\
273 & Amph & up \\
159006 & AC159006.2 & up \\
2974 & Gucy1b2 & up \\
375387 & Nrros & up \\
69391 & 1700018A14Rik & up \\
102637973 & Gm34653 & up \\
79679 & Vtcn1 & up \\
43242 & Gm43242 & up \\
\hline
\end{tabular}


Table 2. Top ten genes demonstrated by connectivity degree in the PPI network

\begin{tabular}{lll}
\hline Gene Symbol & Gene title & Degree \\
\hline POLR2F & RNA polymerase II, I and III subunit F & 13 \\
HIST1H2BJ & Histone cluster 1, H2bj & 11 \\
PPP1CC & Protein phosphatase 1 catalytic subunit & 11 \\
& gamma & 10 \\
HOXB4 & Homeobox B4 & 10 \\
ARG1 & Arginase 1 & 10 \\
APITD1 & Apoptosis-inducing, TAF9-like domain 1 & 10 \\
BUB1B & BUB1 mitotic checkpoint serine/threonine & 10 \\
& kinase B & 10 \\
POLR2K & RNA polymerase II, I and III subunit K & 9 \\
HOXC4 & Homeobox C4 & 9 \\
HOXB3 & Homeobox B3 & 9 \\
\hline
\end{tabular}

